\title{
Fragmentation barriers of toroidal and bubble nuclei
}

\author{
H. M. Xu, C. A. Gagliardi, and R. E. Tribble \\ Cyclotron Institute, Texas A\&M University, College Station, Texas 77843 \\ C. Y. Wong \\ Physics Division, Oak Ridge National Laboratory, Oak Ridge, Tennessee 37831
}

(Received 5 November 1993)

\begin{abstract}
With an improved Boltzmann-Uehling-Uhlenbeck model, the deformation energy surfaces in reactions leading to the formation of toroidal and bubble nuclei are examined for central ${ }^{92} \mathrm{Mo}+{ }^{92} \mathrm{Mo}$ collisions. We found that the potential maximum, or the fragmentation barrier, occurs at times close to the times when these exotic shapes are formed. However, due to the dynamics of large amplitude compression and expansion, the fragmentation barriers are significantly higher than those estimated from the liquid-drop models.
\end{abstract}

PACS number(s): 25.70.Pq, 21.65.+f, 24.10.Cn

Recently, exotic nuclear shapes with toroidal and bubble topologies, analogous to those studied some time ago [1-4], were observed and studied extensively in microscopic transport models [5-17]. Though these transport models have proved to be very useful in predicting the reaction dynamics of the earlier stages which lead to the formation of these exotic shapes, such models cannot, nonetheless, provide reliable information on multiparticle observables such as mass and charge yields or multiplicity distributions of the final fragments. This arises mainly from the lack of higher-order multiparticle correlations and fluctuations which could be important for the later decay stages of the reaction. [Most of current microscopic models, such as the Boltzmann-Uehling-Uhlenbeck (BUU), incorporated only one-body + two-body interactions.] On the other hand, statistical models, such as transition-state models $[18,19]$, the finite-range model [20], as well as multiparticle phase-space models [21-23], have proved to be very powerful in describing late stages of the reactions. In particular, results from such models have been used successfully in interpreting exclusive experimental data in fission and multifragmentation processes. The major parameters in the transition-state or finite-range models are the barrier heights for fission or fragmentation which play important roles in determining the branching ratios of various fragment yields. In contrast, multiparticle phase-space models usually consider the phase space volumes and statistical weights of var- ious fragment configurations for a system expanded to very low densities [21-23].

The purpose of this work is to compare the configurations of the system when the toroidal or bubble nuclei are formed, with the assumptions used in the standard statistical models. In particular, we investigate, from a dynamical (time-dependent) view, the following two key issues which are important in making connections to statistical models: (1) whether the barriers are reached at the times when the exotic shapes are formed; and (2) whether the low density assumption assumed in the fragmentation models is valid for studying the decay of these exotic shapes. For this purpose, we have calculated, with an improved BUU model [15], the deformation energy surface following the dynamics of entrance channels leading to the formation of these exotic shapes. We demonstrate that the potential maximum, or the fragmentation barrier, indeed occurs at times close to the times when these exotic shapes are formed. We find that these exotic shapes are formed when the systems expand to very low densities. However, due to the dynamics of large amplitude compression and expansion which are responsible for the formation of these exotic shapes, the fragmentation barriers are significantly higher than those estimated recently from liquid-drop models $[24,25]$.

We simulate the dynamics of nucleus-nucleus collisions with the Boltzmann-Uehling-Uhlenbeck equation [27]

$$
\frac{\partial f_{1}}{\partial t}+\mathbf{v} \cdot \nabla_{r} f_{1}-\nabla_{r} U \cdot \nabla_{p} f_{1}=\frac{4}{(2 \pi)^{3}} \int d^{3} k_{2} d \Omega \frac{d \sigma_{n n}}{d \Omega} v_{12}\left[f_{3} f_{4}\left(1-f_{1}\right)\left(1-f_{2}\right)-f_{1} f_{2}\left(1-f_{3}\right)\left(1-f_{4}\right)\right]
$$

with the lattice Hamiltonian method of Lenk and Pandharipande [28]. This method provides a reasonable nuclear surface and accurate energy conservation, thus allowing the potential energy surface for the formation of toroidal and bubble nuclei to be evaluated. In Eq. (1), $d \sigma_{n n} / d \Omega$ is the in-medium cross section, $v_{12}$ is the relative velocity for the colliding nucleons, and $U$ is the total mean-field potential consisting of the Coulomb potential and a nuclear potential with isoscalar and symme- try terms [26]. In our calculations, we use two parameter sets [27] for the equation of motion (EOS) which correspond to values of nuclear compressibility $K=200 \mathrm{MeV}$ (soft EOS) and $K=375 \mathrm{MeV}$ (stiff EOS), respectively. For simplicity, $d \sigma_{n n} / d \Omega$ is chosen to be isotropic and independent of energy [27]. The mean-field and the Pauliblocking factors in the collision integral are averaged over an ensemble of 200 parallel simulations.

Examples of our calculations are displayed in Figs. 1 
and 2 for ${ }^{92} \mathrm{Mo}+{ }^{92} \mathrm{Mo}$ collisions at $E / A=75 \mathrm{MeV}$ and $b=0$, for both the stiff (Fig. 1) and the soft (Fig. 2) equations of state. Clearly, for calculations with the stiff EOS, a toroidal nucleus is formed at time $t \approx 120 \mathrm{fm} / c$ with its symmetrical axis parallel to the beam direction. This torus is unstable and eventually breaks up simultaneously into three clusters of nearly equal sizes in a coplanar fashion. In contrast, in the calculations with the soft EOS (see Fig. 2), a nuclear bubble appears to emerge which eventually breaks up into clusters in a volumelike fashion (clusters are emitted approximately isotropically). From both Fig. 1 and Fig. 2, it is easy to see that the dynamics of compression and expansion play a very important role in forming these exotic shapes.
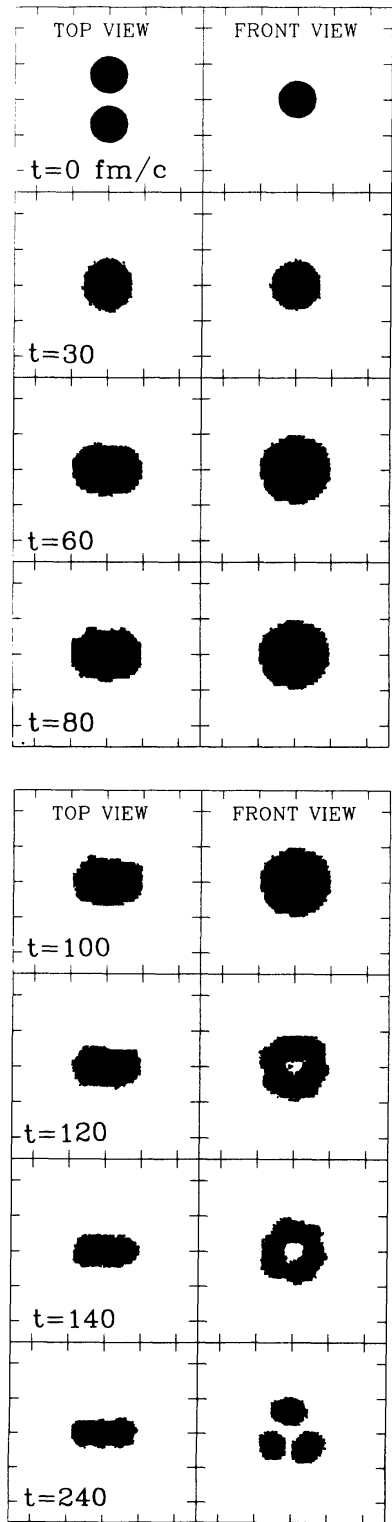

FIG. 1. BUU calculations with the stiff EOS for ${ }^{92} \mathrm{Mo}+{ }^{92} \mathrm{Mo}$ collisions at $E / A=75 \mathrm{MeV}$ and $b=0$. Only regions with densities $\rho \geq 0.1 \rho_{0}$ are shown. The scales between neighboring ticks are $10 \mathrm{fm}$.
To see this more quantitatively, we show in the top panel of Fig. 3 the evolution of the average density, defined as $\langle\rho\rangle=\int_{D} \rho^{2} d^{3} r /\left(\int_{D} \rho d^{3} r\right)$ [29], as a function of time. Here, $D$ indicates the regions with $\rho \geq 0.1 \rho_{0}$, where $\rho_{0}$ is the nuclear saturation density. The corresponding residue masses are shown in the bottom panel. The residue mass curves exhibit two distinct tendencies: a rapid decrease, resembling the preequilibrium emission at earlier times, and a gradual decrease, resembling slow evaporation of particles, at later times. The compressionexpansion dynamics is clearly illustrated in the top panel: for calculations with both the stiff EOS (solid line) and the soft EOS (dashed line), a maximum average density of about $\langle\rho\rangle_{\max } \approx 1.2\left\langle\rho_{0}\right\rangle$ is reached quite early at $t \approx$ 25-30 fm/c. Following this modest compression, significant expansion is developed at later times with minimum densities $\langle\rho\rangle_{\min } \approx 0.2\left\langle\rho_{0}\right\rangle$, which is reached at $t \approx 100$
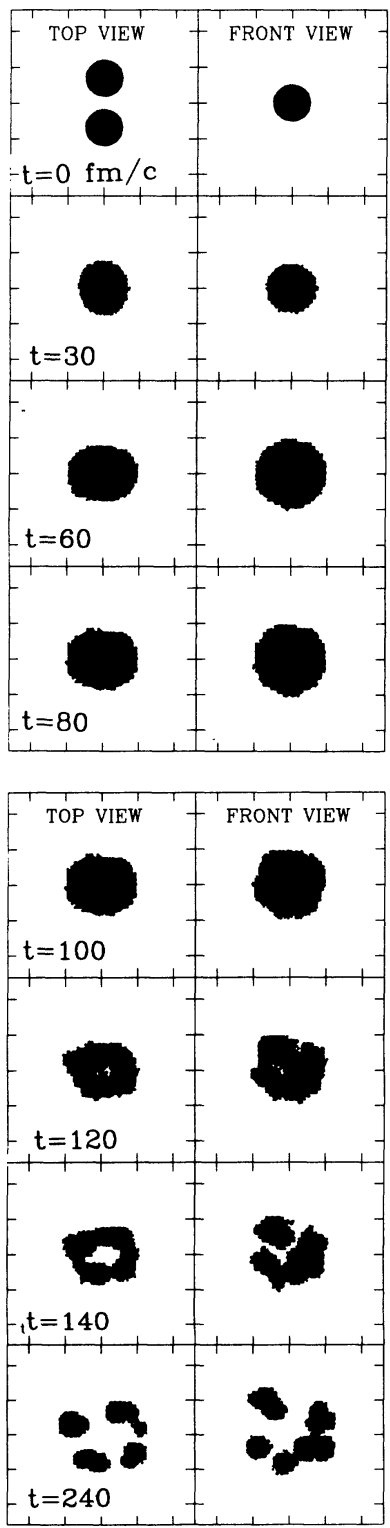

FIG. 2. Same as Fig. 1, but calculated with the soft EOS. 


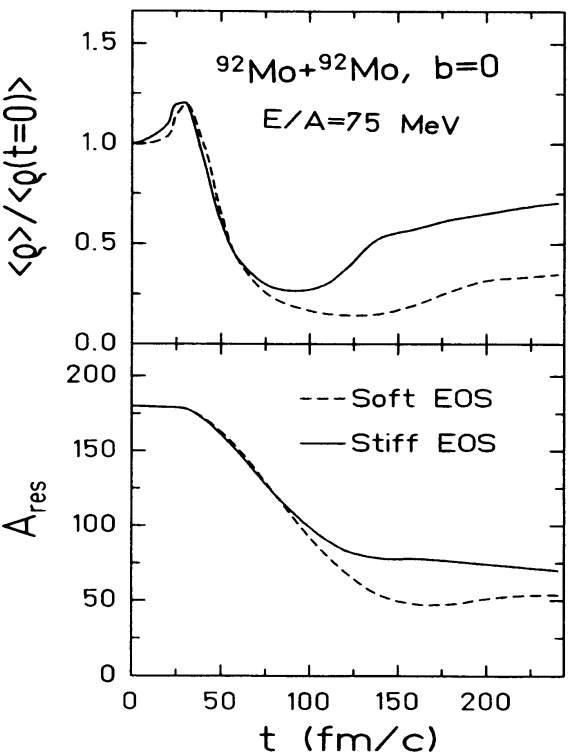

FIG. 3. Top panel: the average density as a function of time for both the stiff EOS (solid line) and the soft EOS (dashed line); bottom panel: the corresponding residue mass as a function of time.

$\mathrm{fm} / c$. The average densities gradually increase at later times, $t \gtrsim 100 \mathrm{fm} / c$, reflecting the condensation into individual fragments. Though densities obtained for both equations of state at early times are about the same, calculations with the stiff EOS (solid lines) have slightly higher densities at later times than those calculated with the soft EOS. This is because the stiff EOS has a larger surface tension strength, and thus the tendency for the residues to expand or break up is less. (See also Figs. 1, 2, which show that calculations with soft EOS tend to have a larger number of clusters in the final stages.) We note in Fig. 3 that quite low densities are reached at the times $t \approx 100 \mathrm{fm} / c$, which are close to the times when the toroidal and bubble geometries are formed (see Figs. 1, 2). The occurrence of low densities with exotic shapes coincides with the low freeze-out density assumption used in the statistical fragmentation models $[22,23]$. Nonetheless, such low densities have not been considered in estimating the multifission or multifragmentation barriers based on liquid drop models $[24,25]$. While the effects of compression or expansion can be ignored in low energy processes, such effects clearly cannot be ignored in processes which lead to the formation of the toroidal and bubble nuclei at high energies.

To assess qualitatively the influence of compression and expansion on the values of the barrier height, we follow the dynamics of BUU calculations and estimate the deformation energy as a function of time as follows

$$
E_{\mathrm{def}}^{*}=E_{\text {int }}(T=0, \rho)-E_{\text {int }}\left(T=0, \rho=\rho_{0}\right) .
$$

The second term in the equation is the internal energy for a ground-state nucleus having the same mass; the first term, $E_{\text {int }}(T=0, \rho)$, is the internal energy of a cold nucleus with temperature $T=0$ and density $\rho$. It includes both the kinetic energy due to the Fermi motion and the potential energy [30],

$$
E_{\text {int }}(T=0, \rho)=\int[\tau(\vec{r})+v(\vec{r})] d^{3} r .
$$

In the Thomas-Fermi limit, the Fermi energy density, $\tau(\vec{r})$, has the form:

$$
\tau(\vec{r})=\frac{3}{10}\left(3 \pi^{2}\right)^{2 / 3} \frac{\hbar^{2}}{m}\left[\rho_{p}^{5 / 3}(\vec{r})+\rho_{n}^{5 / 3}(\vec{r})\right] .
$$

The potential energy density $v(\vec{r})$ includes both nuclear and Coulomb interactions. From the above equations, it is clear that the deformation energy, $E_{\text {def }}^{*}$, represents not only the energy changes due to shape transitions considered previously in liquid-drop models [24,25], but also those due to the density changes observed in our BUU calculations.

The remaining excitation energies, in the form of kinetic energies, can be decomposed approximately into the collective excitation energy and thermal excitation energy. (For comparison, in transition-state models $[18,19]$, all remaining excitation energy above the barrier is considered as thermal.) To minimize the contributions from the remainders of the relative kinetic energy due to the initial motion between the projectile and target, we consider here only the transverse components. Thus, following Ref. [30], the collective energy can be estimated by

$$
E_{\mathrm{coll}}^{*}=\frac{1}{2} m \int_{D} \frac{\vec{j}_{\perp}^{2}(\vec{r})}{\rho(\vec{r})} d^{3} r
$$

where

$$
\rho(\vec{r})=\frac{4}{(2 \pi \hbar)^{3}} \int_{D} f(\vec{r}, \vec{p}, t) d^{3} p
$$

is the local density, and

$$
\vec{j}_{\perp}(\vec{r})=\frac{4}{(2 \pi \hbar)^{3}} \int_{D} \frac{\vec{p}_{\perp}}{m} f(\vec{r}, \vec{p}, t) d^{3} p,
$$

is the local collective velocity field. For collisions at the impact parameter $b=0$, there is no rotational energy and therefore $E_{\text {coll }}^{*}$ reflects mainly the outward radial collective energy at later expansion stages. Once the collective energy is estimated, the thermal energy, which characterizes the random component of the excitation energy, can be simply estimated by

$$
E_{\mathrm{the}}^{*}=E_{\perp}-E_{\text {coll }}^{*}-\frac{2}{3} E_{\text {int }}(T=0, \rho) .
$$

Here, $E_{\perp}$, the total transverse energy of the system, consists of the transverse kinetic energy and $\frac{2}{3}$ of the potential energy.

Figure 4 shows, respectively, the deformation energy (top panel), the transverse thermal energy (center), and the collective energy (bottom) as a function of time for residues with local density $\rho \geq 0.1 \rho_{0}$. Since the residue mass decreases continuously by emitting nucleons, the 


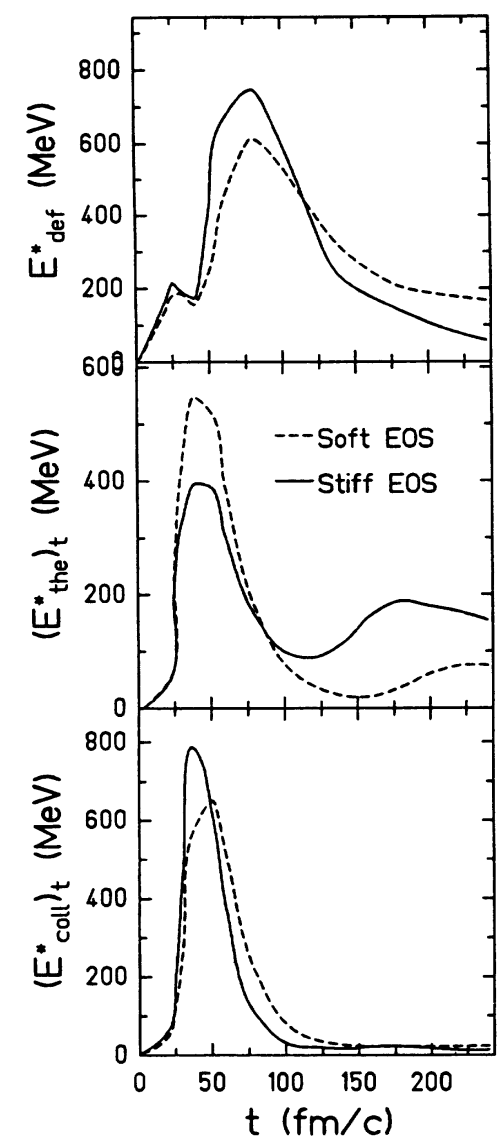

FIG. 4. The components of the excitation energy for ${ }^{92} \mathrm{Mo}+{ }^{92} \mathrm{Mo}$ collisions at $E / A=75 \mathrm{MeV}$ and $b=0$ with both the stiff (solid lines) and the soft (dashed lines) EOS. Top panel: the deformation energy; Central panel: the thermal energy; bottom panel: collective energy. For details, see the text.

corresponding quantities per nucleon are shown in Fig. 5. As exhibited in both figures, both the collective energies, $E_{\text {coll }}^{*}$ and $E_{\text {coll }}^{*} / A_{\text {res }}$ (bottom panels), and the thermal energies, $E_{\text {the }}^{*}$ and $E_{\text {the }}^{*} / A_{\text {res }}$ (central panels), reach maximum values at early times $t \approx 50 \mathrm{fm} / c$. As a result, the systems expand and the deformation energies, both $E_{\text {def }}^{*}$ and $E_{\text {def }}^{*} / A_{\text {res }}$ (top panels), reach maximum values at slightly later times. It is clear from both figures that surprisingly large values of barrier heights, $E_{\text {def }}^{*} \sim 600 \mathrm{MeV}$ for the soft EOS, and $E_{\text {def }}^{*} \sim 750 \mathrm{MeV}$ for the stiff EOS, (or $E_{\mathrm{def}}^{*} / A_{\text {res }} \sim 4 \mathrm{MeV}$ per nucleon for both equations of state), are obtained. They occur at times $t \approx 120-130$ $\mathrm{fm} / c$ for the soft EOS and at $t \approx 80-100 \mathrm{fm} / c$ for the stiff EOS (Fig. 5), which are close to the times when the toroidal or bubbles are already formed (see Figs. 1 and 2). At these times, the average densities of these exotic shapes are near their minimum values (see Fig. 3). Thus, because of the formation of these exotic shapes at very low densities, the barrier heights for fragmentation are significantly high: two to three times higher than those estimated from liquid-drop models [24,25]. On the other hand, since the barrier height depends on the density compression and expansion, which, in turn, depends on the incident energy, they are always exceeded for a

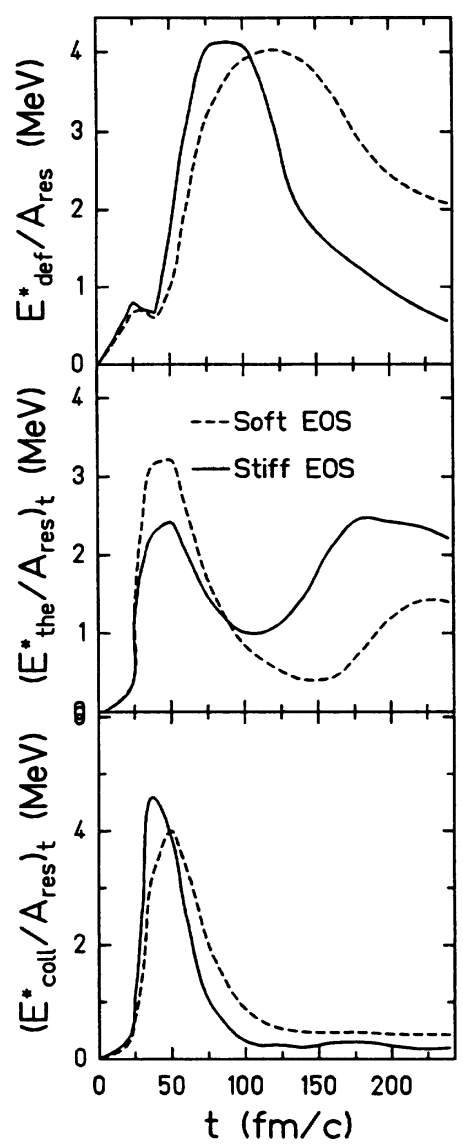

FIG. 5. Same as Fig. 4, but in terms of excitation energy per residue nucleon.

given energy. Lowering the incident energy would always reduce the barrier height, until very low energies for which the effects of compression and expansion become negligible. For these low energy cases, the barrier heights are dominated by effects of nuclear shapes or temperatures as estimated from liquid-drop models [24,25]. Thus at low incident energies, there is an absolute barrier height to overcome which depends on the shape and the temperature. As the incident energy increases, the temperature increases which tends to reduce the barrier height. On the other hand, when the compression and expansion set in, the barrier height increases substantially due to the increase of the compression and expansion. The fact that these barrier heights are reached at times comparable to the times for which the toroidal or bubble nuclei are well-developed indicates that the decay of such exotic shapes could readily be studied within the transition-state models $[18,19]$, provided the fragmentation barrier heights are properly modified to include the effects of expansion. Since the toroidal and bubble nuclei are formed at very low densities, their decay can also be easily incorporated in the multiparticle phase space models [21-23] which assume very low densities. In fact, at the times when these exotic shapes are formed $(t \approx 100$ $\mathrm{fm} / c$ ), most of the remaining excitation energies are in the form of thermal energies (central panels, Figs. 4, 5) with negligible energies in the form of collective excitations (bottom panels), which are neglected in the statis- 
tical models. The gradual increase in the thermal excitation energy at later times reflects the breakup and condensation into individual clusters seen in Figs. 1 and 2. Although the exotic nuclei are formed only at high energies, the thermal excitation energies at the barriers are very low; e.g., for stiff EOS, $\left(E_{\text {the }}^{*} / A_{\text {res }}\right)_{t} \approx 1 \mathrm{MeV}$ for these incident energies, because (1) significant amounts of mass and energy are taken away by the emission of particles before the system reaches the barriers; and (2) significant amounts of energy are stored in the form of potential energy $E_{\text {def }}^{*}$, which raises the barrier heights significantly. These low thermal excitations explain why the final clusters are nearly equal as observed in our BUU model ( see Figs. 1 and 2).

In summary, with an improved BUU model which accurately conserves energy, we have followed the reaction dynamics and estimated the fragmentation barriers for the formation of toroidal and bubble nuclei. We find that these exotic shapes are formed at low densities because of the effects of large amplitude compression and expansion. As a result of this compression and expansion, the barrier heights for these exotic nuclei are significantly higher than those estimated from liquid-drop models. Our results demonstrate that the decay of these exotic shapes can be readily incorporated in the standard statistical models such as the transition-state models $[18,19]$ or the multiparticle phase-space models [21-23].

This work was supported in part by the U.S. DOE under Grant No. DE-FG05-86ER40256 and Contract No. DE-AC05-84OR21400 managed by Martin Marietta Energy Systems, Inc, and the Robert A. Welch Foundation.
[1] J. A. Wheeler, Nucleonics Notebook, 1950, unpublished.

[2] P. Siemens and H. Bethe, Phys. Rev. Lett. 18, 704 (1967).

[3] C.Y. Wong, Phys. Lett. 41B, 446 (1972); 41B, 451 (1972); K.T.R. Davies et al., ibid. 41B, 455 (1972); C.Y. Wong, Ann. Phys. 77, 279 (1973).

[4] C.Y. Wong et al., Phys. Lett. 66B, 19 (1977).

[5] G. Bertsch and D. Mundinger, Phys. Rev. C 17, 1646 (1978).

[6] J. Lattimer et al., Nucl. Phys. A432, 646 (1985).

[7] H. Reinhardt et al., Nucl. Phys. A432, 630 (1985).

[8] J. Németh, M. Barranco, C. Ngô, and E. Tomasi, Z. Phys. A 320, 691 (1985); J. Németh, M. Barranco, J. Desbois, and C. Ngô, ibid. 323, 419 (1986); 325, 347 (1986).

[9] D. Vautherin, J. Treiner, and M. Vénéroni, Phys. Lett. B 191, 6 (1987).

[10] L. Vinet et al., Phys. Lett. B 172, 17 (1986).

[11] L.G. Moretto et al., Phys. Rev. Lett. 69, 1884 (1992).

[12] W. Bauer et al., Phys. Rev. Lett. 69, 1888 (1992).

[13] D.H.E. Gross et al., Ann. Phys. 1, 467 (1992).

[14] B. Borderie et al., Phys. Lett. B 302, 15 (1993).

[15] H.M. Xu et al., Phys. Rev. C 48, 933 (1993); H.M. Xu et al., Nucl. Phys. A (in press); H.M. Xu, Phys. Rev. C 46, R2144 (1992).

[16] G. Batko and J. Randrup, Nucl. Phys. A563, 97 (1993).

[17] A. Bonasera, N. Colonna, and F. Gulminelli, INFN-LNS report, 1992 (unpublished).

[18] L.G. Moretto, Phys. Lett. 40B, 185 (1972); Nucl. Phys. A247, 211 (1975).

[19] J.A. Lopez and J. Randrup, Nucl. Phys. A503, 183 (1989).

[20] A.J. Sierk, Phys. Rev. C 33, 2039 (1986); Phys. Rev. Lett. 55, 582 (1985).

[21] S.E. Koonin and J. Randrup, Nucl. Phys. A356, 223 (1981); A474, 173 (1987); G. Fai and J. Randrup, Nucl. Phys. A381, 557 (1982); A404, 551 (1983); Phys. Lett. 115B, 281 (1982).

[22] D.H.E. Gross, Rep. Prog. Phys. 53, 605 (1990); B.H. Sa, Y.M. Zheng, and X.Z. Zhang, Int. J. Mod. Phys. A 5, 843 (1990).

[23] J.P. Bondorf et al., Nucl. Phys. A443, 321 (1985); Nucl. Phys. A444, 460 (1985).

[24] F. Haddad and G. Royer, J. Phys. G 18, L153 (1992).

[25] G.X. Dai et al., Texas A\&M University Report No. 93-07 (unpublished).

[26] H.M. Xu et al., Phys. Rev. Lett. 65, 843 (1990).

[27] G.F. Bertsch and S. Das Gupta, Phys. Rep. 160, 189 (1988), and references therein.

[28] R.J. Lenk and V.R. Pandharipande, Phys. Rev. C 39, 2242 (1989).

[29] E. Suraud et al., Phys. Lett. B 229, 359 (1989).

[30] B. Remaud et al., Nucl. Phys. A488, 423c (1988). 